\title{
Cambios en la diversidad funcional del sotobosque y la tasa de descomposición frente a diferentes intensidades de uso silvopastoril en el noroeste de la Patagonia, Argentina
}

\author{
Juliana E. Arias Sepúlveda ${ }^{1, \otimes}$ \& Verónica Chillo ${ }^{2 / 3}$ \\ ${ }^{1}$ Universidad del Aconcagua, Ciclo de Licenciaturas "San Pedro Nolasco", Mendoza, Argentina. ${ }^{2}$ CONICET. ${ }^{3}$ Instituto de \\ InvestigacionesenRecursosNaturales, Agroecologíay DesarrolloRural(IRNAD), SedeAndina, UniversidadNacionaldeRío
} Negro. El Bolsón, Río Negro, Argentina.

\begin{abstract}
RESUMEN. La pérdida de diversidad funcional por cambios en el uso del suelo es clave para alcanzar un manejo sostenible ya que cumple un papel fundamental en el funcionamiento del ecosistema al influir directamente en procesos ecológicos. En los bosques andino-patagónicos, la ganadería es una de las principales actividades productivas que genera cambios en los ecosistemas naturales. El objetivo del trabajo fue caracterizar la variación de la diversidad funcional de la vegetación del sotobosque en sitios con distintas intensidades de uso silvopastoril, y los efectos que estas variaciones producen sobre la tasa de descomposición del mantillo, en bosques mixtos de ciprés y coihue del suroeste de la Provincia de Río Negro (Argentina). Para ello se caracterizó la variación en las propiedades funcionales de la vegetación (i.e., ciclo y forma de vida, arquitectura, hábitat, tamaño y textura de la hoja, sistema radical) y se cuantificó la tasa de descomposición del mantillo del sitio. Los resultados mostraron que tanto el índice de diversidad funcional $\left(\mathrm{FD}_{\mathrm{O}}\right)$ como la tasa de descomposición fueron mayores en los sitios con alta intensidad de uso silvopastoril, pero no se encontró una relación significativa entre ambas variables. También se detectó que ciertos rasgos funcionales de las plantas (e.g., la arquitectura roseta, el hábitat postrado y la forma de vida helecho) tienen un efecto significativo sobre el porcentaje de peso perdido del mantillo. En sitios con alta intensidad de uso silvopastoril aumentó la proporción relativa de cobertura de especies con arquitectura roseta y hábitat postrado, lo que derivó en un aumento de la tasa de descomposición. Esto indica que la actividad silvopastoril podría estar modificando la comunidad original hacia especies de rápida adquisición de recursos, alterando a su vez al ciclado de nutrientes. En este sentido, el funcionamiento del ecosistema estaría influenciado por la presencia de rasgos funcionales de mayor importancia, en vez de la diversidad funcional en sí misma.
\end{abstract}

[Palabras clave: bosques mixtos, características funcionales, pastoreo, biodiversidad]

Aвstract. Changes in plant functional diversity and decomposition rate under different silvopastoral use intensities in northwest Patagonia, Argentina. Functional diversity loss by human land-use changes is of great importance for achieving sustainable management, as it plays a key role in ecosystem functioning through a direct influence over ecological processes. In northwest Patagonian forests, livestock production is among the main activities affecting natural ecosystems. The aim of the study was to characterize changes in functional diversity of understory vegetation in sites with different silvopastoral use intensity, and the effects that these variations may have on rate of litter decomposition in mixed forests of cypress and coihue of southwest Río Negro Province, Argentina. Changes in plant functional traits (life cycle, life style, architecture, habitat, leaf size, leaf texture and root system) were characterized, and the decomposition rate of each site's litter was quantified. Our results showed that both functional diversity index $\left(\mathrm{FD}_{\mathrm{O}}\right)$ and decomposition rate were higher under high silvopastoral use intensity, but the relationship was not significant. Results also showed that certain plant functional traits (i.e., rosette architecture, prostrate habitat and fern life form) have a significant effect on litter lost weight. Under high silvopastoral use intensity, the relative proportion of species with rosette architecture and prostrate habitat increases, which led to an increase in decomposition rate. This indicates that silvopastoral activity may modify the original community composition towards species with a rapid resource acquisition, altering also nutrient cycling. In this sense, ecosystem functioning seems to be influenced by certain functional traits, rather than functional diversity itself.

[Keywords: mixed forests, functional traits, livestock grazing, biodiversity]

Editor asociado: Guillermo Martinez Pastur

$\bowtie$ julianaeas2411@gmail.com 


\section{INTRODUCCIÓN}

Los cambios en el uso del suelo, la pérdida y la fragmentación del hábitat, y los intercambios bióticos están produciendo una reducción acelerada de la biodiversidad (genética, funcional y de paisajes) en todo el mundo (Newbold et al. 2015). La diversidad funcional es entendida como el valor, el rango y la abundancia relativa de características o rasgos de una comunidad que tienen un determinado efecto sobre el ecosistema (Díaz and Cabido 2001). Este tipo de diversidad cumple un papel clave en el funcionamiento de los ecosistemas dado que influye de manera directa sobre los procesos ecológicos y determina las respuestas de los organismos ante las presiones del medio. Por lo tanto, la pérdida de diversidad funcional tendrá un impacto directo en el funcionamiento de los procesos ecosistémicos (e.g., ciclo de nutrientes y producción primaria neta) (Díaz et al. 2007a; de Bello et al. 2010).

Las distintas estrategias de la vegetación para la adquisición y la conservación de los recursos disponibles determinan la calidad y la cantidad de biomasa aportada al mantillo; esto influye sobre la tasa de descomposición, un proceso clave en el ciclado de nutrientes (Díaz et al. 2007a; Cornwell et al. 2008). Por ejemplo, las especies con ciclo de vida anual aportan biomasa al mantillo a una mayor velocidad que las especies con ciclos de vida perennes. Las especies con crecimiento secundario (leñosas), hojas coriáceas o compuestos secundarios presentan una tasa de descomposición más lenta debido al mayor contenido de lignina y compuestos carbonados complejos (Cornwell et al. 2008), lo que, a su vez, mejora su tolerancia a la herbivoría (e.g., hojas coriáceas) (Díaz et al. 2007b).

La ganadería mediante el uso silvopastoril de los bosques templados andino-patagónicos es una de las principales actividades productivas. Esta práctica consiste en la apertura del dosel mediante raleos, así como la eliminación de árboles muertos en pie y la recolección de leña. Esto permite un mayor ingreso de la luz y las precipitaciones y favorece el crecimiento de las plantas del sotobosque para la alimentación del ganado (Somlo et al. 1997; Peri et al. 2016). En este sentido, la composición de la comunidad vegetal del sotobosque está determinada no sólo por la presión de herbivoría, sino también por la disponibilidad mayor de los recursos (e.g., luz y agua en el suelo). Estudios recientes (Peri et al. 2016) resaltan la importancia de considerar de forma integrada la respuesta de todo el ecosistema, tanto al manejo ganadero (carga ganadera) cómo a la práctica silvícola (apertura de dosel y raleo); los resultados pueden ser antagónicos según la intensidad de ambas actividades, consideradas por separado. El manejo silvopastoril es considerado una alternativa productiva viable para la región en térmicos económicos, ecológicos y sociales, lo que permite la diversificación dentro de las unidades productivas. Sin embargo, los estudios se han centrado en unas pocos tipos forestales y condiciones ecológicas (e.g., bosques de Nothofagus antarctica) y son escasos los estudios de los impactos que el uso silvopastoril puede tener sobre los diferentes tipos forestales a lo largo de la Patagonia.

En los bosques mixtos de ciprés de la cordillera (Austrocedrus chilensis (D. Don) Flor. et Boult) y coihue (Nothofagus dombeyi (Mirb.) Blume) del noroeste de la Patagonia, la producción de ganado bovino se centra en la cría y engorde (entre 0.2 y $0.1 \mathrm{UG} / \mathrm{ha}$ ), con escasas pautas de manejo del rodeo y baja inversión en infraestructura rural (Cardozo and Reuque 2012). La influencia de estas prácticas sobre el bosque puede producir impactos negativos a través de cambios en la composición de la vegetación, lo cual puede, a su vez, alterar las interacciones que caracterizan a la comunidad (e.g., se ha reportado un aumento de la riqueza vegetal del sotobosque debido a un incremento del establecimiento y dispersión de especies exóticas) (Blackhall et al. 2008; Piazza et al. 2016). Los cambios en la composición de la comunidad en sitios con ganado están dados por una mayor cobertura de hierbas y gramíneas (Raffaele et al. 2011; Piazza et al. 2016). En estos tipos forestales, los árboles dominantes (ciprés y coihue) y acompañantes (Maytenusboaria,Schinuspatagonicusy Lomatia hirsuta) son siempreverdes, por lo que su aporte a la biomasa del mantillo no es tan contundente como en bosques de Nothofagus caducifolios. Esto resalta la importancia de considerar los efectos que la vegetación del sotobosque puede tener sobre los procesos ecológicos de interés, en especial en sitios de alto uso silvopastoril donde la cobertura de dosel es baja (Amoroso et al. 2015).

Estos cambios en la composición de la comunidad implican alteraciones en las características funcionales, que podrían afectar el ciclado de nutrientes (Relva et al. 2014) y la productividad primaria neta (Lencinas et al. 2011). Sin embargo, poco se sabe acerca de los efectos que dichos cambios producen 
sobre el funcionamiento del ecosistema. El objetivo de este trabajo fue caracterizar los cambios en la diversidad funcional de la vegetación del sotobosque dados por dos intensidades de uso silvopastoril, y los efectos que esos cambios producen en la tasa de descomposición de cada sitio. Se estableció como hipótesis que en bosques mixtos de ciprés y coihue del noroeste de la Patagonia, la actividad silvopastoril genera cambios en la composición de la comunidad vegetal del sotobosque aumentando la dominancia de características de efecto funcional relacionadas con la adquisición y conservación de recursos. Esos cambios podrían impactar sobre la tasa de descomposición del mantillo de cada sitio. Se predice que un aumento en la intensidad de uso silvopastoril generará una comunidad vegetal dominada por características que permitan la adquisición rápida de los recursos (e.g., ciclo de vida anual y plantas perennes, herbáceas de arquitectura roseta, hojas medianas a grandes y sistema radical lateral). Se prevé que este tipo de comunidades producirá un mantillo con una tasa mayor de descomposición que bosques con menor intensidad de uso silvopastoril.

\section{Materiales y MÉTOdos}

El estudio se llevó a cabo en el suroeste de

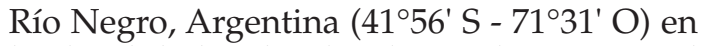
las localidades de El Bolsón, El Manso y El Foyel. El clima es templado-húmedo a fríohúmedo, con fuertes nevadas en el invierno y heladas casi todo el año (Cabrera 1976). La temperatura media anual varía entre 4 y $14^{\circ} \mathrm{C}$, muy influenciada por la altitud (Dimitri 1972). Los bosques seleccionados se corresponden a bosques mixtos de ciprés ( $A$. chilensis) y coihue ( $N$. dombeyi) entre las isohietas de 1600 y 2000 mm/año, y que se caracterizan por presentar una alta cobertura del dosel $(>90 \%)$ con árboles que alcanzan los $30 \mathrm{~m}$ de altura (Dimitri 1972).

Se trabajó en establecimientos de productores forestales ganaderos con predominio de bosque mixto de ciprés de la cordillera y coihue, y con dos niveles de uso silvopastoril en función del impacto generado por el sistema de uso tradicional. El sistema tradicional de ganadería bovina consiste en la permanencia y el pastoreo en las partes bajas de los valles durante otoño, invierno y primavera (invernada) y el movimiento hacia lengales y ñirantales de mayor altitud durante el verano (veranada). El pastoreo y la permanencia del ganado en las partes bajas se logran a través de la apertura del dosel para mejorar el forraje y el uso maderero. En este contexto se genera una mayor intensidad en los bosques abiertos (cobertura de coihue entre $\sim 20 \%$ y $30 \%$ ) y aledaños, y una menor intensidad de uso en sectores alejados de los anteriores (cobertura de coihue entre $\sim 40 \%$ y $60 \%$ ). De esta manera se generan dos intensidades de uso silvopastoril contrastantes dentro de un mismo tipo de bosque con similar estructura, composición, historia de uso e historia de disturbios naturales que se definió en función de la cobertura de heces observada en el campo $(4.5 \pm 3.1 \%$ en los sitios de alta y $0.47 \pm 0.06 \%$ en los sitios de baja intensidad de uso silvopastoril).

Se trabajó con un diseño de muestreo apareado con el objetivo de reducir la variabilidad debida a la historia de uso (e.g., cargas ganaderas históricas) y los potenciales factores ambientales (e.g., sequías, incendios leves, etc.). Se seleccionaron cinco sitios de muestreo ubicados en establecimientos agropecuarios diferentes (dos en El Bolsón, dos en El Manso y uno en El Foyel). Cada sitio de muestreo incluyó dos sectores separados entre sí por una distancia mínima de 500 $\mathrm{m}$, que incluían dos rodales con diferentes usos silvopastoriles en un diseño apareado: a) rodales con alto, y b) bajo uso ganadero $(\mathrm{n}=5$ establecimientos $\times 2$ sectores $\times 2$ tratamientos).

En cada rodal se cuantificó la cobertura por especie del sotobosque en 6 transectas de $2 \times 10$ $\mathrm{m}$, separadas $50 \mathrm{~m}$ entre sí, entre noviembre y diciembrede2013. Cada transecta se subdividió en cinco cuadrantes consecutivos de $2 \times 2 \mathrm{~m}$. En cada cuadrante se determinó visualmente el porcentaje de cobertura por proyección de copa de cada especie vegetal de $<1.5 \mathrm{~m}$ de altura (sotobosque). La estimación siempre fue realizada por el mismo observador para evitar errores dados por la subjetividad del método. Dado que cada especie puede cubrir hasta 100\% del cuadrante y que la cobertura estimada es una suma de la cobertura de todas las especies, el valor de cobertura de cada cuadrante puede asumir valores entre 0 e . Para los análisis estadísticos posteriores, este valor absoluto fue relativizado dentro de cada transecta. Asimismo, se cuantificó el diámetro a la altura del pecho (DAP) de todos los individuos de ciprés y coihue de más de 1.5 $m$ de altura dentro de cada transecta, y luego se estimó área basal por hectárea para cada rodal para estimar el grado de ocupación de la masa forestal. También se estimó de forma 
visual el porcentaje de cobertura de heces frescas (e.g., sin coloración blanca) de vacas en cada cuadrante.

Por otro lado, se cuantificó la tasa de descomposición del mantillo de cada rodal por medio de la técnica de bolsas, propuesta por Pérez-Harguindeguy et al. (2008). Durante el otoño (mayo 2013) se colectaron hojas senescentes frescas de las plantas dominantes de sotobosque y del dosel forestal de cada rodal. Las hojas de los árboles fueron colectadas mediante la instalación de una red de $1 \mathrm{~m}^{2}$ a $0.5 \mathrm{~m}$ de altura desde el suelo, activa durante una semana. Las hojas senescentes de la vegetación del sotobosque fueron colectadas a mano, por debajo y alrededor (área máxima de $25 \mathrm{~m}^{2}$ ) de la red de recolección de hojas de dosel. Las hojas colectadas fueron secadas en estufa $\left(60^{\circ} \mathrm{C}\right)$ hasta peso constante, y la mezcla de hojas senescentes de cada rodal fue utilizada para armar las bolsas de descomposición. De esta forma se obtiene una mezcla de broza con composición estimada similar a la que caracteriza el rodal, lo que permite asociar la composición de la vegetación con la tasa de descomposición (Garnier et al. 2007; Laliberté and Tylianakis 2012). Dichas bolsas $(10 \times 10 \mathrm{~cm}$ con red de $2 \mathrm{~mm}$ de poro) contuvieron entre 2 y $3 \mathrm{~g}$ de mezcla de hojas senescentes de cada rodal (compuesta por $50 \%$ del sotobosque y $50 \%$ del dosel), y fueron dispuestas bajo el dosel mixto de un ciprés y un coihue (a $1 \mathrm{~m}$ de la base del árbol dominante) para evitar variaciones dadas por la identidad de de las especies de dosel (Vivanco and Austin 2008). El objetivo de esta técnica fue cuantificar la tasa de descomposición de cada rodal, teniendo en cuenta las diferencias en la composición de la vegetación y no en las características microambientales, por lo que las bolsas se ubicaron siempre debajo de la misma cobertura de copa. Sin embargo, las diferencias en la apertura de dosel entre intensidades de uso silvopastoril genera diferencias en las condiciones microclimáticas que puede influir sobre el proceso de descomposición. En cada rodal se colocaron un total de 4 bolsas (sub-muestras), la mitad fueron colectadas a los 6 meses de incubación y la otra mitad luego de 12 meses de incubación. Una vez colectadas se llevaron a laboratorio, donde se secaron a $60{ }^{\circ} \mathrm{C}$ en horno hasta obtener un peso constante. Concluido el secado, las muestras se pesaron en balanza de precisión (0.001 g) y se sometieron a un proceso de incineración en mufla $\left(500^{\circ} \mathrm{C}\right)$ para eliminar del cálculo del peso seco perdido la contaminación por material orgánico externo a la muestra (Vivanco and Austin 2008).
Las características de efecto funcional de la vegetación fueron seleccionadas buscando aquellas que se relacionen directa o indirectamente con la capacidad de descomposición de la hoja (ciclo y forma de vida, tamaño y textura de hoja) o las características de micrositio que modifiquen la capacidad de descomposición de la hoja (i.e., arquitectura, hábitat, sistema radical) (Tabla 1 ), acorde a lo propuesto en numerosos trabajos (de Bello et al. 2006; Garnier et al. 2007; Pérez-Harguindegy et al. 2013). Se buscaron características blandas (e.g., ciclo y forma de vida, textura de hoja), que son aquellas que a pesar de no tener una relación tan fuerte o directa con la tasa de descomposición como las características duras (e.g., contenido de nitrógeno en hoja) son fácilmente cuantificables de forma indirecta. Esto permite su identificación en campo sin necesidad de realizar costos análisis químicos (Pérez-Harguindegy et al. 2013). Luego, se identificaron las mismas en las especies utilizando la historia de vida de las mismas en base a la bibliografía, el herbario elaborado durante el muestreo y herbarios digitales.

Con estos datos se calculó la diversidad funcional mediante el índice de Rao $\left(\mathrm{FD}_{\mathrm{Q}}\right)$ (Botta-Dukat 2005):

$$
\mathrm{FD}_{\mathrm{Q}}=\sum_{i=1}^{S-1} \sum_{j=i+1}^{S} d_{i j} p_{i} p_{j}
$$

Donde $\mathrm{S}=$ valor máximo de especies de la comunidad, $\mathrm{p}=$ frecuencia relativa, $\mathrm{d}=$ diferencia en la característica funcional entre la especie i y la especie j, y FD = diferencia promedio entre dos individuos elegidos al azar. Se calculó un valor de $\mathrm{FD}_{\mathrm{O}}$ para cada sitio de alta y baja intensidad de uso silvopastoril.

Para evaluar si existían diferencias en las variables estructurales (área basal por hectárea y porcentaje de cobertura de heces) entre las dos intensidades de uso silvopastoril, realizamos un análisis de componentes principales (PCA) (Legendre and Legendre 2012). Por otra parte, para analizar cómo varía la diversidad funcional de la vegetación del sotobosque entre intensidad de uso silvopastoril se aplicó la prueba $t$ o de Student (Zar 1999) al índice de diversidad funcional $\left(\mathrm{FD}_{\mathrm{Q}}\right)$. Se utilizó esta prueba estadística dado que, en base a un concepto de parsimonia, es el análisis más sencillo para comparar dos tratamientos $y$, por lo tanto, presenta menor error estadístico 
Tabla 1. Características de efecto funcional y sus categorías.

Table 1. Functional effect traits and its categories.

\begin{tabular}{ll}
\hline \multicolumn{1}{c}{ Característica } & \multicolumn{1}{c}{ Categorías } \\
\hline Ciclo de vida & Anual \\
& Decidua \\
& Perenne \\
Forma de vida & Helecho \\
& Gramínea \\
& Herbácea \\
& Arbusto \\
& Árbol \\
Tamaño de la hoja & Pequeña $(<2 \mathrm{~cm})$ \\
& Mediana $(2-5 \mathrm{~cm})$ \\
& Grande $(>5 \mathrm{~cm})$ \\
Textura de la hoja & Membranosa \\
& Intermedia \\
& Coriácea \\
Arquitectura & Roseta \\
& Colchón \\
& Estolonífero \\
& Varias ramas \\
& Tallo principal \\
Hábitat & Postrado \\
& Trepadora \\
Sistema radical & Erecto \\
& Lateral \\
& Pivotante \\
\hline
\end{tabular}

Nota: basado en características utilizadas por de Bello et al. (2006).

de tipo II. Para caracterizar la variación en las características funcionales de la vegetación en sitios con alta y baja intensidad de uso silvopastoril se realizó un análisis de varianza (ANOVA) corregido para un número elevado de comparaciones, y se utilizó la suma de la cobertura relativa de especies por sitio para cada característica (Zar 1999). Para comparar la tasa de descomposición entre intensidad de uso silvopastoril, se llevó a cabo una prueba de $t$ para las muestras incubadas durante 6 meses y otro para las muestras de 12 meses (Zar 1999). Para analizar la relación entre la tasa de descomposición y la diversidad funcional de la vegetación se realizó una prueba de correlación de Pearson. Posteriormente, se correlacionó el índice de diversidad funcional $\left(\mathrm{FD}_{\mathrm{O}}\right)$ con el porcentaje de peso perdido de mantillo para los periodos de incubación de 6 y 12 meses. Finalmente, se seleccionaron aquellas categorías funcionales que presentaban diferencias significativas entre los sitios de alta y baja intensidad de uso silvopastoril y se correlacionaron con el porcentaje de peso perdido de mantillo en ambos períodos de incubación. Todos los análisis se llevaron a cabo utilizando el software R (R Development Team 2014).

\section{Resultados}

El análisis de las variables estructurales presentó diferencias entre las dos intensidades de uso silvopastoril (Figura 1). Se puede apreciar la separación de los sitios de estudio en dos grupos de acuerdo a los diferentes valores de área basal por hectárea y porcentaje de cobertura de heces. La comparación de la diversidad funcional entre sitios de alta y baja intensidad de uso silvopastoril mostró diferencias significativas $(t=2.741 ; P=0.018)$, siendo mayor el valor de $\mathrm{FD}_{\mathrm{Q}}$ en sitios de alta intensidad de uso silvopastoril (Figura 2). Por otro lado, el análisis de la variación en las características funcionales de la vegetación del sotobosque entre sitios de alta y baja intensidad de uso silvopastoril reveló diferencias significativas en todas las categorías referidas al ciclo de vida (Tabla 2). En las características referidas a forma de vida, textura de la hoja, arquitectura y hábitat se encontraron diferencias significativas en algunas de sus categorías, mientras que el resto de las características (sistema radical, tamaño de la hoja) no presentaron diferencias significativas entre intensidades de uso silvopastoril (Tabla 2).

La tasa de descomposición mostró diferencias significativas entre las distintas intensidades de uso silvopastoril, tanto para las muestras incubadas durante 6 meses ( $t=3.733 ; P=0.002$ ) como para las incubadas durante 12 meses

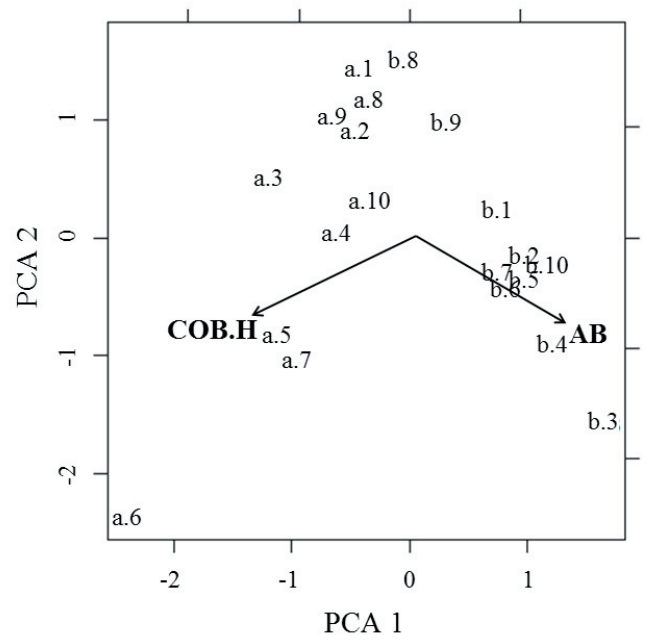

Figura 1. Análisis de componentes principales (PCA) entre los sitios de estudio, en función del área basal por hectárea (AB) y del porcentaje de cobertura de heces (COB.H), a.n: sitios con alto uso silvopastoril, b.n: sitios con bajo uso silvopastoril, $\mathrm{n}=10$. El primer eje (PCA 1) explica 78\% de la varianza acumulada.

Figure 1. Principal component analysis (PCA) between study sites based on basal area per hectare $(\mathrm{AB})$ and the percentage of feces cover (COB.H), a.n: sites with high silvopastoral use, b.n: sites with low silvopastoral use, $\mathrm{n}=10$. First axis (PCA 1) explains $78 \%$ of the variance. 


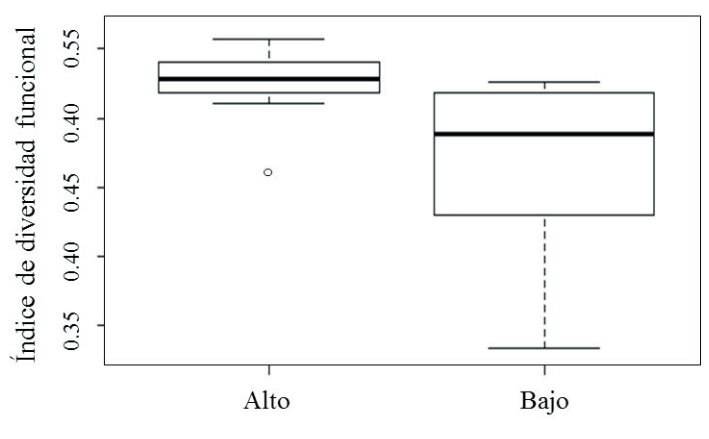

Intensidad de uso silvopastoril

Figura 2. Índice de diversidad funcional $\left(\mathrm{FD}_{\mathrm{O}}\right)$ de sitios con alta y baja intensidad de uso silvopastoril. Las partes superior e inferior de la caja muestran el tercer cuartil y el primer cuartil de los datos respectivamente, la línea central representa la mediana, las líneas verticales que se extienden desde la mitad de la caja indican la dispersión de los datos, y los puntos muestran datos atípicos.

Figure 2. Functional diversity index ( $\mathrm{FD}_{\mathrm{O}}$ ) of sites with high and low silvopastoral use intensity. Top and bottom parts of the box are third and first quartile of the data, central line is the median vertical lines expanding outside the box are data dispersion, and the point represents outlier data.

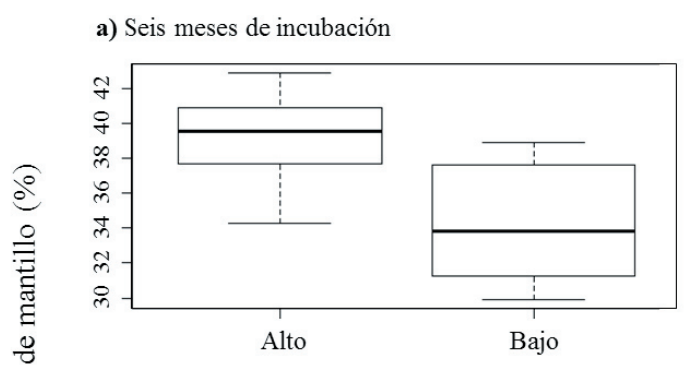

b) Doce meses de incubación

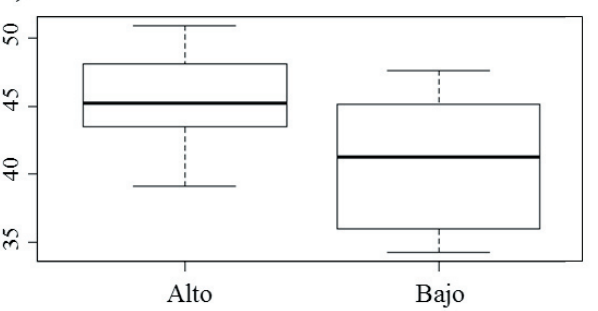

Intensidad de uso silvopastoril

Figura 3. Porcentaje de peso perdido del mantillo en sitios con alta y baja intensidad de uso silvopastoril en los períodos de incubación de 6 y 12 meses, respectivamente. Las partes superior e inferior de la caja muestran el tercer cuartil y el primer cuartil de los datos, respectivamente, la línea central representa la mediana, las líneas verticales que se extienden desde la mitad de la caja indican la dispersión de los datos, y los puntos muestran datos atípicos.

Figure 3. Percentage of litter lost weight in sites with high and low silvopastoral use intensity, in 6 months and 12 months incubation periods. Top and bottom part of the box are third and first quartile of the data, central line is the median vertical lines expanding outside the box are data dispersion, and the points represent outlier data.
Tabla 2. Análisis de varianza (ANOVA) de una vía entre las características funcionales de la vegetación del sotobosque (promedio de la proporción relativa de cobertura) y la intensidad de uso silvopastoril (alta y baja). El asterisco marca las categorías con diferencias significativas $(P<0.05)$.

Table 2. One way analysis of variance (ANOVA) between understory vegetation functional traits (average of the relative proportion of coverage) and silvopastoral use intensity (high and low). Categories with significant differences are marked with an asterisk $(P<0.05)$.

\begin{tabular}{|c|c|c|c|c|}
\hline Características & $\overline{\text { Alta }}$ & Baja & $F$ & $P$ \\
\hline \multicolumn{5}{|l|}{ Ciclo de vida } \\
\hline Anual $^{*}$ & 0.109 & 0.052 & 12.73 & 0.002 \\
\hline Decidua* & 0.057 & 0.024 & 6.94 & 0.017 \\
\hline Perenne* & 0.834 & 0.924 & 15.69 & 0.000 \\
\hline \multicolumn{5}{|l|}{ Forma de vida } \\
\hline Helecho* & 0.018 & 0.067 & 8.083 & 0.011 \\
\hline Gramínea* & 0.168 & 0.091 & 12.29 & 0.002 \\
\hline Herbácea & 0.393 & 0.297 & 3.738 & 0.069 \\
\hline Arbusto & 0.254 & 0.301 & 1.958 & 0.179 \\
\hline Árbol & 0.167 & 0.244 & 1.578 & 0.225 \\
\hline \multicolumn{5}{|l|}{ Tamaño de la hoja } \\
\hline Sin hojas & 0.000 & 0.000 & 0.092 & 0.766 \\
\hline Pequeñas & 0.323 & 0.382 & 0.788 & 0.386 \\
\hline Medianas & 0.303 & 0.308 & 0.016 & 0.900 \\
\hline Grandes & 0.374 & 0.309 & 2.470 & 0.133 \\
\hline \multicolumn{5}{|l|}{ Textura de la hoja } \\
\hline Sin hojas & 0.000 & 0.000 & 0.092 & 0.766 \\
\hline Membranosa & 0.219 & 0.170 & 1.579 & 0.225 \\
\hline Intermedia* & 0.426 & 0.315 & 7.576 & 0.013 \\
\hline Coriácea* & 0.355 & 0.515 & 6.417 & 0.021 \\
\hline \multicolumn{5}{|l|}{ Arquitectura } \\
\hline Roseta ${ }^{*}$ & 0.192 & 0.034 & 32.580 & 0.000 \\
\hline Colchón & 0.057 & 0.033 & 3.312 & 0.086 \\
\hline Estolonífero & 0.256 & 0.311 & 2.436 & 0.136 \\
\hline Varias ramas & 0.139 & 0.132 & 0.084 & 0.775 \\
\hline Tallo principal ${ }^{*}$ & 0.355 & 0.489 & 6.335 & 0.022 \\
\hline \multicolumn{5}{|l|}{ Hábitat } \\
\hline Postrado* & 0.151 & 0.074 & 14.300 & 0.001 \\
\hline Trepadora & 0.051 & 0.069 & 1.894 & 0.186 \\
\hline Erecto* & 0.799 & 0.857 & 5.092 & 0.037 \\
\hline \multicolumn{5}{|l|}{ Sistema radical } \\
\hline Lateral & 0.289 & 0.267 & 0.246 & 0.626 \\
\hline Pivotante & 0.711 & 0.733 & 0.246 & 0.626 \\
\hline
\end{tabular}

$(t=2.563 ; P=0.020)$. En ambos periodos de incubación, los sitios con alta intensidad de uso silvopastoril mostraron mayor porcentaje de peso perdido que los sitios con baja intensidad de uso silvopastoril (Figura 3). Se halló una correlación marginal y no significativa entre $\mathrm{FD}_{\mathrm{O}} \mathrm{y}$ el porcentaje de peso perdido del mantillo en ambos periodos de incubación $(\mathrm{r}=0.108, P=0.65$ para 6 meses; $\mathrm{r}=0.034, P=0.88$ para 12 meses) (Figura 4 ).

Para las categorías seleccionadas de cada característica funcional, la que presentó la relación más fuerte y significativa (negativa) con el porcentaje de peso perdido del mantillo fue la categoría arquitectura roseta $(\mathrm{r}=0.63$, $P=0.002$ para 6 meses; $\mathrm{r}=0.55, P=0.012$ para 12 meses). La categoría hábitat postrado presentó una relación positiva significativa 


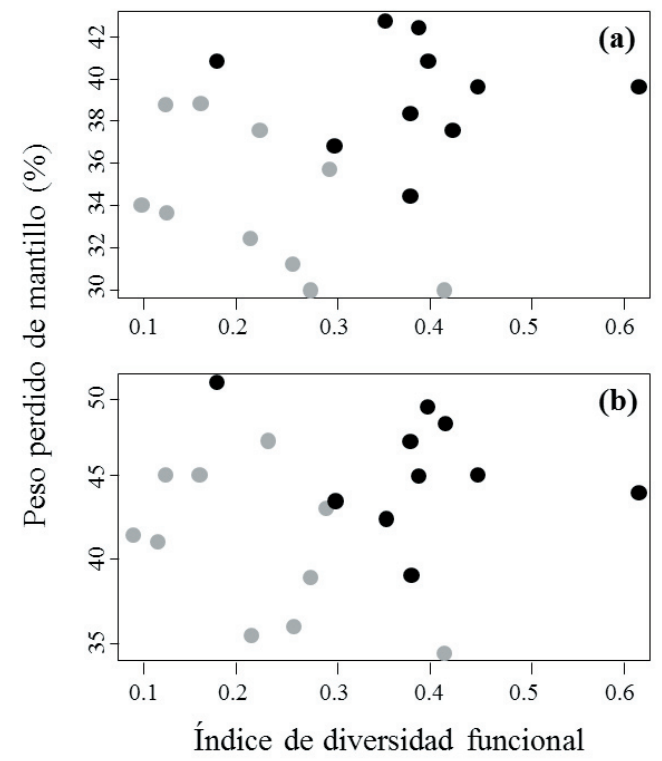

Figura 4. Correlación de Pearson entre el índice de diversidad funcional y el porcentaje de peso perdido del mantillo a los 6 meses de incubación ( $\mathrm{a}, \mathrm{r}=0.108, \mathrm{P}=0.65$ ) y a los 12 meses de incubación ( $\mathrm{b}, \mathrm{r}=0.034, \mathrm{P}=0.88$ ). Los símbolos grises representan sitios de baja intensidad de uso silvopastoril, mientras que los sitios de alta de intensidad de uso silvopastoril están representados en color negro. Se muestra el valor del coeficiente de correlación de Pearson en cada período de incubación, así como su significancia $(P<0.05)$.

Figure 4. Pearson's correlation between functional diversity index and the percentage of litter lost weight at 6 months of incubation $(\mathrm{a}, \mathrm{r}=0.108, \mathrm{P}=0.65)$ and 12 months of incubation $(\mathrm{b}, \mathrm{r}=0.034, \mathrm{P}=0.88)$. The gray symbols represent low silvopastoral use intensity sites, while sites high silvopastoral use intensity are represented in black color. The value of Pearson correlation coefficient for each incubation period and its significance is displayed $(P<0.05)$.

con el porcentaje de peso perdido del mantillo para ambos períodos de incubación $(\mathrm{r}=0.47$, $P=0.04$ para 6 meses; $r=0.44, P=0.05$ para 12 meses). La categoría forma de vida helecho también mostró una relación con respecto al porcentaje de peso perdido del mantillo, siendo significativa (negativa) sólo en el periodo de 6 meses de incubación $(\mathrm{r}=0.5 ; P=0.03)$. El resto de las categorías (ciclo de vida anual, deciduo y perenne, forma de vida gramínea, textura de hoja intermedia y coriácea, arquitectura de tallo principal y hábitat erecto) no mostraron una relación significativa con el porcentaje de peso perdido del mantillo en los periodos de incubación de 6 y 12 meses.

\section{DISCUSIÓN}

\section{Efecto del uso silvopastoril sobre la diversidad funcional}

Existen numerosas evidencias de que los disturbios comunes en la Patagonia, como los incendios, las invasiones biológicas y el pastoreo (Gowda et al. 2011), generan cambios en la composición, la estructura y la dinámica de la vegetación, lo cual altera la diversidad (Veblen et al. 2008; Relva et al. 2010; González et al. 2014). En los bosques de lenga (Nothofagus pumilio) del sur de la Patagonia se ha evidenciado un aumento de la diversidad vegetal después de la cosecha silvícola, debido principalmente a un aumento en la abundancia de especies exóticas y de especies anuales e intolerantes a la sombra, así como a la extinción local de especies tolerantes a la sombra (Lencinas et al. 2011). En bosques de ñire bajo manejo silvícola y silvopastoril, donde la apertura del dosel alcanza o supera el 50\%, se reportó un bajo efecto sobre la riqueza, pero un efecto mucho mayor sobre la composición y la cobertura de plantas del sotobosque, con una dominancia de hierbas y pastos anuales (Peri et al. 2016). Por otro lado, un estudio reciente (Piazza et al. 2016) demuestra que el disturbio por ganadería en bosques de coihue del noroeste de la Patagonia aumenta la riqueza de especies y genera un cambio en la comunidad hacia la pérdida local de arbustos nativos y el aumento de la cobertura de herbáceas. Sin embargo, la relación entre dichos cambios en la diversidad y las consecuencias sobre el funcionamiento del ecosistema ha sido poco estudiada.

En este trabajo se analizaron por primera vez los efectos que distintas intensidades de uso silvopastoril tienen sobre la diversidad funcional y su relación con el proceso de descomposición en los bosques mixtos de ciprés de la cordillera y coihue del noroeste de la Patagonia. Los resultados del presente estudio muestran que el aumento en la intensidad de uso silvopastoril genera cambios en la diversidad funcional de la comunidad vegetal del sotobosque. Se esperaba encontrar una menor diversidad funcional en los sitios de mayor intensidad de uso silvopastoril debido a que otros trabajos han reportado que en los bosques bajo estudio el pastoreo por ganado doméstico y por grandes ungulados disminuye la diversidad de la vegetación (Vázquez 2002; Blackhall et al. 2008). Sin embargo, los resultados muestran que en sitios de alta intensidad de uso silvopastoril, el valor de diversidad funcional $\left(\mathrm{FD}_{\mathrm{Q}}\right)$ fue mayor que en sitios de baja intensidad de uso. Esto podría deberse a que la apertura del dosel genera un incremento en la disponibilidad de luz. Tanto en bosques de lenga como en bosques de ñire del sur de la Patagonia se ha encontrado 
que los cambios microclimáticos generados por una mayor radiación (por apertura de dosel) pueden producir un aumento de la diversidad del sotobosque, debido a una colonización rápida de especies intolerantes a la sombra y con ciclos de vida anual (Lencinas et al. 2011; Soler et al. 2013; Peri et al. 2016) y, probablemente, una distribución más equitativa de las características funcionales.

Existe un modelo teórico ampliamente demostrado por resultados empíricos, que propone que las presiones que ejercen los disturbios determinan distintas estrategias en relación a la inversión en los recursos en la vegetación. Estas estrategias de inversión de recursos varían entre una adquisición rápida de carbono y nutrientes, y una lenta adquisición de estos nutrientes con su conservación posterior dentro de tejidos bien protegidos (Westoby and Wright 2006). En general, se espera que el aumento en la cantidad de luz favorezca la dominancia de plantas de crecimiento rápido pero corta duración, caracterizadas por hojas de vida corta y alto contenido de nitrógeno, consideradas baratas en términos de uso de C (Poorter and Bongers 2006). Por otro lado, se esperaba que el pastoreo favoreciera a las plantas con características que les permitan tolerar las presiones (como un ciclo de vida anual en el que la planta invierte en un rápido crecimiento y reproducción, o un hábitat postrado y arquitectura estolonífera y roseta que le permitan a la planta escapar al bocado del ganado) (Díaz et al. 2007b). Los resultados de este estudio coinciden con este modelo teórico ya que los sitios de alta intensidad de uso silvopastoril (menor cobertura de dosel y mayor presencia de ganado) están caracterizados por plantas con ciclo de vida anual y deciduo, hábitat postrado y arquitectura roseta. Mientras tanto, los sitios con baja intensidad de uso silvopastoril (dosel más cerrado y menos rastros de presencia de ganado) están caracterizados por plantas con ciclo de vida perenne, hábitat erecto y arquitectura de tallo principal. Esto significa que la comunidad cambia hacia una estrategia de rápida adquisición de recursos, respuesta probablemente dada por la dominancia del factor luz sobre el factor pastoreo (Cingolani et al. 2005). Otros autores reportaron resultados similares para sitios con pastoreo por grandes ungulados y con distintos grados de apertura de dosel, dados por mortalidad de árboles dominantes en bosques del noroeste de la Patagonia (Blackhall et al. 2008; Relva et al. 2008).

\section{Relación entre la diversidad funcional y la tasa de descomposición}

Los resultados permiten acepar la hipótesis: la tasa de descomposición de sitios de alta intensidad de uso silvopastoril fue mayor que en sitios de baja intensidad de uso. En función del modelo del espectro económico de la hoja, el dominio de plantas con estrategias de rápida adquisición de recursos en sitios de alta intensidad de uso silvopastoril puede estar relacionado con una mayor tasa de descomposición (Cornwell et al. 2008). Se muestra que las categorías con una influencia significativa en la descomposición se corresponden con hojas coriáceas, forma de vida helecho, arquitectura roseta y hábitat postrado. Las hojas coriáceas presentan un mayor contenido de lignina y compuestos secundarios, por lo que tendrán un proceso de descomposición de menor velocidad (de Paz et al. 2014). La forma de vida helecho por lo general presenta un ciclo de vida perenne y hojas con textura coriácea, lo que conlleva a una descomposición lenta debido al aporte lento de hojarasca con un alto contenido en lignina y compuestos secundarios al mantillo (Cornwell et al. 2008; de Paz et al. 2014). Las plantas con arquitectura roseta y hábitat postrado poseen hojas de textura membranosa o intermedia y ramas finas; también tienen menos lignina y compuestos secundarios, por lo que se descomponen con más rapidez (de Paz et al. 2014). Estos resultados son consistentes con trabajos publicados en otras partes del mundo, que establecen que aquellas plantas con menor contenido de lignina y mayor contenido de compuestos que promuevan una alta activad fisiológica y crecimiento tendrán una descomposición rápida (Chapín 2003; Sayer 2006).

Para entender el papel de la diversidad funcional sobre el funcionamiento de los ecosistemas se han propuesto dos explicaciones alternativas. Por un lado, el efecto de selección establece que a mayor riqueza de especies en una comunidad, mayores probabilidades de que en esa comunidad estén presentes especies con rasgos particulares importantes que dominarán el funcionamiento del ecosistema (Díaz and Cabido 2001; Loreau 2010). Por ejemplo, la presencia de árboles con estructuras leñosas complejas y extensos sistemas radicales tiene efectos sobre la retención de sedimentos y absorción y retención de carbono (Díaz and Cabido 2001). Por otro lado, el efecto de complementariedad de nichos establece que a mayor diversidad aumentan los rasgos funcionales representados en una comunidad, y esto proporciona mayores oportunidades de 
usar más eficientemente los recursos, en un entorno espacial o temporalmente variable (Díaz and Cabido 2001; Loreau 2010) (e.g., en zonas áridas, un incremento en la diversidad de especies vegetales aumenta la productividad primaria a través de la ocurrencia de especies que realizan un uso complementario del recurso hídrico) (de Bello et al. 2006).

A pesar de que numerosos modelos teóricos y antecedentes empíricos en el mundo han demostrado que un aumento de la diversidad promueve un mejor funcionamiento del ecosistema (Gamfeldt et al. 2008; Isbell et al. 2011), los resultados de este trabajo muestran que el índice de diversidad funcional $\left(\mathrm{FD}_{\mathrm{Q}}\right)$ no tiene una relación directa con la tasa de descomposición. Esto significa que los cambios en la diversidad de características funcionales representadas en la población no estarían influyendo en el funcionamiento del ecosistema. Sin embargo, los resultados de este trabajo sí demuestran que ciertos rasgos funcionales (como la arquitectura roseta, el hábitat postrado y la forma de vida helecho) tienen una relación significativa con la tasa de descomposición del mantillo. Así, el funcionamiento del ecosistema estaría influenciado por la presencia de rasgos funcionales de mayor importancia, de acuerdo con la explicación del efecto de selección. En varias partes del mundo se reportaron resultados similares, lo que demuestra que dicho mecanismo predomina entre diversas comunidades vegetales (Cardinale et al. 2006; Díaz et al. 2007a; Mokany et al. 2008).

Agradecimientos. Carolina Trigo, Julie Powel, Ezequiel Villacide, Mariano Amoroso, Carlos Rezzano y Diego P. Vázquez colaboraron con el diseño del estudio y el trabajo de campo. Guillermo Martínez Pastur y dos revisores anónimos contribuyeron a mejorar notablemente el manuscrito. Agradecemos especialmente a Roberto Criado, Lisandro y Oscar Lanfré y Estancia el Foyel por dejarnos trabajar en sus establecimientos. El trabajo fue parcialmente financiado por una beca posdoctoral Fundación Bunge y Born, por Rufford Small Grants Foundation y por la Universidad Nacional de Río Negro (PI 40B-311).

\section{REFERENCIAS}

Amoroso, M. M., V. Chillo, C. A. Rezzano, V. Alcalá, and E. Villacide. 2015. Efecto del manejo silvopastoril sobre la estructura demográfica del dosel y la composición del sotobosque en bosques mixtos de ciprés de la cordillera (Austrocedus chilensis) y coihue (Nothofagus dombeyi). V Congreso Latinoamericano de Agroecología, La Plata, Buenos Aires, Argentina.

Blackhall, M., E. Raffaele, and T. T. Veblen. 2008. Cattle affect early post-fire regeneration in a Nothofagus dombeyiAustrocedrus chilensis mixed forest in northern Patagonia, Argentina. Biological Conservation 141:2251-2261.

de Bello, F., J. Lepš, and M. T. Sebastià. 2006. Variations in species and functional plant diversity along climatic and grazing gradients. Ecography 29:801-810.

de Bello, F., S. Lavorel, S. Díaz, R. Harrington, J. H. C. Cornelissen, et al. 2010. Towards an assessment of multiple ecosystem processes and services via functional traits. Biodivers Conserv 19:2873-2893.

Botta-Dukát, Z. 2005. Rao's quadratic entropy as a measure of functional diversity based on multiple traits. Journal of Vegetation Science 16:533-540.

Cabrera, A. L. 1976. Regiones Fitogeográficas Argentinas, Fasciculo 1. Enciclopedia Argentina de Agricultura y Jardinería. 2da ed., tomo II.

Cardinale, B., D. S. Srivastava, J. E. Duffy, J. P. Wright, A. L. Downing, et al. 2006. Effects of biodiversity on the functioning of trophic groups and ecosystems. Nature 443:989-992.

Cardozo, A. G., and R. Reuque. 2012. Caracterización de la producción ganadera correspondiente al sector rionegrino de la Comarca Andina del Paralelo $42^{\circ}$. Comunicación Técnica N ${ }^{\circ} 80$, AER El Bolsón.

Chapin, F. S. 2003. Effects of plant traits on ecosystem and regional processes: a conceptual framework for predicting the consequences of global change. Annals of Botany 91:455-463.

Cingolani, A; Noi Meir, I, and Díaz, S. 2005. Grazing effects on rangeland diversity: a synthesis of contemporary models. Ecological Applications 15:757-773.

Cornwell, W. K., J. H. C. Cornelissen, K. Amatangelo, E. Dorrepaal, V. T. Eviner, et al. 2008. Plant species traits are the predominant control on litter decomposition rates within biomes worldwide. Ecology Letters 11:1065-1071.

Díaz, S., and M. Cabido. 2001. Vive la différence: plant functional diversity matters to ecosystem processes. Ecology and Evolution 16(11):646-655.

Díaz, S., S. Lavorel, F. de Bello, F. Quétier, K. Grigulis, and T. M. Robson. 2007a. Incorporating plant functional diversity effects in ecosystem service assessments. PNAS 104:20684-20689.

Díaz, S., S. Lavorel, S. McIntyre, V. Falczuk, F. Casanoves, et al. 2007b. Plant trait responses to grazing - a global synthesis. Global Change Biology 13:313-341.

Dimitri, M. J. 1972. La Región de los Bosques Andino-Patagónicos, Sinopsis general. Colección científica del INTA, tomo X.

Gamfeldt, L., H. Hillebrand, and P. R. Jonsson. 2008. Multiple functions increase the importance of biodiversity for overall ecosystem functioning. Ecology 89(5):1223-1231. 
Garnier, E., S. Lavorel, P. Ansquer, H. Castro, P. Cruz, et al. 2007. Assessing the Effects of Land-use Change on Plant Traits, Communities and Ecosystem Functioning in Grasslands: A Standardized Methodology and Lessons from an Application to 11 European Sites. Annals of Botany 99:967-985.

González, M. E., M. Amoroso, A. Lara, T. T. Veblen, C. Donoso, et al. 2014. Ecología de disturbios y su influencia en los bosques templados de Chile y Argentina [12]. Pp. 411-502 en: C. Donoso, M. E. González and A. Lara (eds.). Ecología Forestal. Bases para el manejo sustentable y conservación de los bosques nativos de Chile. Ediciones UACh.

Gowda, J. H., T. Kitzberger, and A. C. Premoli. 2011. Landscape responses to a century of land use along the northern Patagonian forest-steppe transition. Plant Ecology 213:259-272.

Isbell, F., V. Calcagno, A. Héctor, J. Connolly, W. S. Harpole, et al. 2011. High plant diversity is needed to maintain ecosystem services. Nature 477:199-203.

Laliberté, E., and J. M. Tylianakis. 2012. Cascading effects of long-term land-use changes on plant traits and ecosystem functioning. Ecology 93(1):145-155.

Legendre, P., and L. Legendre. 2012. Numerical Ecology. Third English Edition. Editorial Elsevier.

Lencinas, M. V., G. Martínez Pastur, E. Gallo, and J. M. Cellini. 2011. Alternative silvicultural practices with variable retention to improve understory plant diversity conservation in southern Patagonian forests. Forest Ecology and Management 262:1236-1250.

Loreau, M. 2010. Linking biodiversity and ecosystems: towards a unifying ecological theory. Philosophical Transactions of the Royal Society B 365:49-60.

Milchunas, D. G., O. E. Sala, and W. K. Lauenroth. 1988. A generalized model of the effects of grazing by large herbivores on grassland community structure. The American Naturalist 132:87-106.

Mokany, K., J. Ash, S. and Roxburgh. 2008. Functional identity is more important than diversity in influencing ecosystem processes in a temperate native grassland. Journal of Ecology 96:884-893.

Newbold, T., L. Hudson, S. L. L. Hill, S. Contu, I. Lysenko, et al. 2015. Global effects of land use on local terrestrial biodiversity. Nature 520:45-50.

de Paz, M., M. E. Gobbi, and E. Raffaele. 2014. Mantillo de las especies leñosas de matorrales del NO de la Patagonia: abundancia, composición, estructura y heterogeneidad. Bol Soc Argent Bot 48(3-4):525-541.

Pérez-Harguindeguy, N., C. M. Blundo, and D. E. Gurvich. 2008. More than the sum of its parts? Assessing litter heterogeneity effects on the decomposition of litter mixtures through leaf chemistry. Plant Soil 303:151-159.

Pérez-Harguindeguy, N., S. Díaz, E. Garnier, S. Lavorel, et al. 2013. New handbook for standardized measurement of plant functional traits worldwide. Australian Journal of Botany 61:167-234.

Peri, P. L., N. E. Hansen, H. A. Bahamonde, M. V. Lencinas, A. R. von Müller, et al. 2016. Silvopastoral Systems under Native Forest in Patagonia Argentina Pp. 117-168 en: P. L. Peri, F. Dube and A. Varela (eds.). Silvopastoral Systems in Southern South America. Springer International Publishing Switzerland.

Piazza, M. V., L. A. Garibaldi, T. Kitzberger, and E. J. Chaneton. 2016. Impact of introduced herbivores on understory vegetation along a regional moisture gradient in Patagonian beech forests. Forest Ecology and Management 366: 11-22.

Poorter, L., and F. Bongers. 2006. Leaf traits are good predictors of plant performance across 53 rain forest species. Ecology 87:1733-1743.

R Development Team. 2014.

Raffaele, E., T. T. Veblen, M. Blackhall, and N. Tercero-Bucardo. 2011. Synergistic influences of introduced herbivores and fire on vegetation change in northern Patagonia, Argentina. Journal of Vegetation Science 22:59-71.

Relva, M. A., C. López Westerholm, and T. Kitzberger. 2008. Effects of introduced ungulates on forest understory communities in northern Patagonia are modified by timing and severity of stand mortality. Plant Ecology 201:1122.

Relva, M. A., M. A. Núñez, and D. Simberloff. 2010. Introduced deer reduce native plant cover and facilitate invasion of non-native tree species: evidence for invasional meltdown. Biological Invasions 12:303-311.

Relva, M. A., E. Castán, and M. J. Mazzarino. 2014. Litter and soil properties are not altered by invasive deer browsing in forests of NW Patagonia. Acta Oecologica 54:45-50.

Sayer, E. J. 2006. Using experimental manipulation to assess the roles of leaf litter in the functioning of forest ecosystems. Biol Rev 81:1-31.

Soler, R., G. Martínez Pastur, P. Peri, M. V. Lencinas, and F. Pulido. 2013. Are silvopastoral systems compatible with forest regeneration? An integrative approach in southern Patagonia. Agroforestry Systems 87(6):1213-1227.

Somlo, R., G. Bonvissuto, T. Schlichter, P. Laclau, P. Peri, and M. Alloggia. 1997. Silvopastoral use of Argentine Patagonian forests [7]. Pp. 237-250 en: A. M. Gordon and S. M. Newman (eds.). Temperate Agroforestry System. Cab International.

Vázquez, D. P. 2002. Multiple effects of introduced mammalian herbivores in a temperate forest. Biological Invasions 4:175-191.

Veblen, T. T., T. Kitzberger, E. Raffaele, M. Mermoz, M. E. González, et al. 2008. The historical range of variability of fires in the Andean-Patagonian Nothofagus forest region. International Journal of Wildland Fire 17:724-741.

Vivanco, L., and A. T. Austin. 2008. Tree species identity alters forest litter decomposition through long-term plant and soil interactions in Patagonia, Argentina. Journal of Ecology 96:727-736.

Westoby, M., and I. J. Wright. 2006. Land-plant ecology on the basis of functional traits. Trends in Ecology and Evolution 21:261-268.

Zar, J. H. 1999. Biostatistical Analysis. Prentice Hall. Cuarta edición 